Preliminary Draft

\title{
Identifying Conventional and Unconventional Monetary Policy Shocks: A Latent Threshold Approach
}

\author{
Takeshi Kimura and Jouchi Nakajima* \\ Bank of Japan, 2-1-1 Nihonbashi, Hongokucho, Chuo-ku, Tokyo 103-8660, JAPAN
}

April 2013

\begin{abstract}
This paper proposes a new estimation framework for identifying monetary policy shocks in both conventional and unconventional policy regimes using a structural VAR model. Exploiting a latent threshold modeling strategy that induces time-varying shrinkage of the parameters, we explore a recursive identification switching with a time-varying overidentification for the interest rate zero lower bound. We empirically analyze Japan's monetary policy to illustrate the proposed approach for modeling regime-switching between conventional and unconventional monetary policy periods, and find that the proposed model is preferred over a nested standard time-varying parameter VAR model. The estimation results show that increasing bank reserves lowers long-term interest rates in the unconventional policy periods, and that the impulse responses of inflation and the output gap to a bank reserve shock appear to be positive but highly uncertain.
\end{abstract}

JEL classification: C32; E52

Keywords: Identification; Latent threshold models; Monetary policy; Time-varying parameter VAR; Zero lower bound

We are grateful for helpful discussions with and comments from Kosuke Aoki, Hiroshi Fujiki, Michael Funke, Takuji Kawamoto, and Mike West, as well as seminar participants at the Bank of Japan, and Hitotsubashi University. The views expressed herein are those of the authors alone and do not necessarily reflect those of the Bank of Japan.

${ }^{*}$ Corresponding author. Tel.: +81-3-3277-2809. Fax: +81-3-5201-6525.

E-mail addresses: takeshi.kimura@boj.or.jp (T. Kimura), jouchi.nakajima@boj.or.jp (J. Nakajima). 


\section{Introduction}

Japan was a front-runner of unconventional monetary policy actions, embarking on a policy of quantitative easing in 2001, nowadays followed by major industrialized countries to tackle the recessionary turbulence after the recent global financial crisis. Against this background, there is a growing literature measuring the quantitative impact of such unconventional policies on the economy (see, e.g., Joyce et al. 2012, for a comprehensive survey). In this context, the present paper proposes a new estimation framework for identifying monetary policy shocks in both conventional and unconventional policy regimes. Central banks in advanced economies face considerable uncertainty regarding the effects of unconventional monetary policy in the unusual economic circumstances following the global financial crisis, and it is typically difficult to identify a purely structural monetary policy shock and isolate the effects of unconventional policy from a number of underlying factors driving the economy. In addition, a fundamental challenge is to estimate the effectiveness of unconventional monetary policy with the relatively short historical data available so far. Given these challenges, there have been a variety of approaches to evaluating the effect of unconventional monetary policy. These can be broadly divided into three strands based on the estimation methodology.

The first approach to evaluating the macroeconomic effects of unconventional monetary policy is the "plug-in" approach, which uses estimates of the impact of unconventional policy measures on asset prices to plug them into standard macroeconomic models. Estimates of the impact on asset prices are provided, among others, by Gagnon et al. (2011) and Wright (2012) focusing on the Federal Reserve's large-scale asset purchases (LSAPs), Joyce et al. (2011) focusing on the Bank of England's asset purchases, and Kimura and Small (2006) and Ueda (2012) focusing on the Bank of Japan's quantitative easing (QE) and other unconventional monetary policy actions. Taken together, these studies suggest that there is considerable empirical evidence of significant changes in asset prices in response to central bank policy measures. In particular, they suggest that these measures have the effect of lowering medium- to long-term bond yields and term premiums. The plug-in approach uses these estimates of the reaction in financial markets as the monetary policy shock associated 
with the unconventional policy actions. By plugging the estimates of the reaction of financial markets into standard macroeconomic models, such as the FRB/US model, the effect of the unconventional monetary policy actions on inflation and output is measured (see, e.g., Chung et al. 2012, and Fuhrer and Olivei 2011). ${ }^{1}$ However, this plug-in approach needs to rely on the estimates of separate studies on the reaction of financial markets; possible biases and uncertainty regarding the estimates, if exist, may lead to unreliable simulation results of the macroeconomic model (see, e.g., Hamilton and $\mathrm{Wu}$ 2012, Bauer and Rudebusch 2011). In addition, unconventional policy shocks are transmitted through a compression of term premiums (the portfolio rebalancing channel) and/or a lowering of expected short-term interest rates (the signaling channel), the effects of which on real activity may differ, as pointed out by Stein (2012), Kiley (2012), and Chen et al. (2012). Regarding the relationship between long-term interest rates and the real economy, however, standard macroeconomic models do not differentiate between these two channels, which may lead to an incorrect assessment of unconventional policy actions. In sum, the problem of the plug-in approach is that the monetary policy shocks are not consistent with the macroeconomic models employed.

The second approach is to employ a structural model using a DSGE model for calibration to examine the separate effects of short-term and long-term interest rates on the real economy. Kiley (2012) and Chen et al. (2012) develop structural models that take financial market segmentation into account and argue that term and risk premiums exert a smaller effect on economic activity than that exerted by short-term interest rates. While it seems to be reasonable to evaluate the effects of unconventional policies using structural models that clearly specify possible transmission channels, the problem is that there are various views on how financial market segmentation should be modeled. For example, Kiley (2012) assumes two types of agents - one that can trade both short- and long-term bonds, and another that can only trade short-term bonds. In contrast, Chen et al. (2012) assume that one agent can trade both bonds but the other can only trade

\footnotetext{
${ }^{1}$ For example, Chung et al. (2012) use the estimate reported in Gagnon et al. (2011) - namely that the Federal Reserve's asset purchases in 2009 lowered the yield on 10-year Treasury and high-grade corporate bonds - to implement simulation exercises employing well-established macroeconomic models such as the FRB/US model. They suggest that the Fed's unconventional policy actions possibly had an effect on macroeconomic variables such as inflation and output.
} 
long-term bonds. Quantitative analyses using such structural models entirely depend on the specified structure, which needs to be carefully examined in light of the real world. Unfortunately, however, we do not yet have sufficient information about which specification best describes the real structure of market segmentation.

Finally, the third approach is a data-driven approach using structural vector autoregression (VAR) with identifying restrictions on the simultaneous relations among variables, where the policy effects and transmission mechanisms are assessed based on impulse response analysis. Studies using this approach include Baumeister and Benati (2010), Iwata (2010), and Kapetanios et al. (2012). Baumeister and Benati (2010), for example, use a time-varying parameter VAR (TVP-VAR) model and attempt to identify monetary policy shocks associated with short-term interest rates in the conventional policy regime as well as those associated with asset purchases in the unconventional policy regime with the interest rate zero lower bound (ZLB). In order to identify the unconventional policy shocks, they define a "pure" spread shock associated with long-term yield spreads, which leaves the short-term rate unchanged for eight quarters, but raises inflation and output growth within a quarter of the impact, i.e., the monetary policy effect arises immediately after the policy actions. ${ }^{2}$ However, such a restriction may not always be plausible when taking the lag structures in monetary policy transmission mechanisms into consideration.

The present paper follows the third approach and seeks to improve on it by proposing a new framework to identify conventional and unconventional monetary policy shocks in the presence of the ZLB in a TVP-VAR model. ${ }^{3}$ Specifically, we incorporate a regime change between conventional and unconventional policies using the "latent threshold model (LTM)" recently proposed by Nakajima and West (2013). The LTM approach seeks to take into account situations in which a parameter process may be significant in some periods but close to zero and practically insignificant in

\footnotetext{
${ }^{2}$ Low or declining yield spreads can be caused not only by asset purchases under the unconventional monetary policy but also by a less vigorous future economy. To extract the former factor only, Baumeister and Benati (2010) impose the sign restriction that the spread compression shock leads to an increase in inflation and output growth within a quarter of the impact.

${ }^{3}$ See, for example, Primiceri (2005) for details on the modeling strategy for TVP-VAR models.
} 
other periods. The purpose underlying the LTM approach is to induce parsimony in modeling, which simplifies high-dimensional time-varying parameters in time series analysis such as the TVP-VAR models. By inducing temporal sparsity in time-varying parameters, the LTM approach helps to reduce estimation uncertainty and improve forecasting performance (see Nakajima and West 2013). This paper utilizes the approach for regime-switching constraints on time-varying parameters associated with the simultaneous effects of structural shocks to identify conventional and unconventional monetary policy shocks. The LTM approach further provides a method for examining whether there are any overidentified structures in the presence of the ZLB. It is reasonable to expect that the interest rate responses to both macroeconomic shocks in the conventional policy regime and bank reserve shocks in the unconventional policy regime are zero when the ZLB is binding. The LTM approach makes it possible to statistically "test" for overidentification at each point in time, i.e., whether the data "prefer" the ZLB structure or not. Incorporating the explicit and implicit assumption of zeros in the time-varying parameter enables us to explore the data-driven overidentified structure without dividing the observation period into subperiods. To empirically assess the approach, we apply it to Japan's monetary policy and economy, gauging the effectiveness of the conventional and unconventional monetary policies.

\section{Methodology}

\subsection{Conventional and unconventional regimes}

In order to identify conventional and unconventional monetary policy shocks, the following simple but fundamental model is considered for each of the regimes (see Table 1). In the conventional policy regime, as shown in Equation (1a), the central bank sets the short-term interest rate $(i)$ as its policy instrument in response to macroeconomic shocks $\left(\varepsilon^{X}\right)$ associated with inflation and the output gap. Deviation from this rule is regarded as a monetary policy shock $\left(\varepsilon^{i}\right)$. In this regime, as shown in Equation (1b), the demand for bank reserves $(m)$ is determined by the short-term interest rate $(i)$ and a precautionary money demand shock $\left(\varepsilon^{d}\right)$. 
Table 1. Identification in conventional and unconventional regimes

\begin{tabular}{|c|c|}
\hline Conventional policy regime & Unconventional policy regime \\
\hline $\begin{array}{l}\text { Interest rate rule } \\
\qquad \text { (1a) } \quad i=\alpha \varepsilon^{X}+\varepsilon^{i}\end{array}$ & $\begin{array}{l}\text { Bank reserves rule } \\
\text { (2a) } \quad m=\beta \varepsilon^{X}+\varepsilon^{m}\end{array}$ \\
\hline $\begin{array}{l}\text { Bank reserves demand function } \\
\text { (1b) } \begin{aligned} m & =-\gamma i+\varepsilon^{d} \\
& =-\gamma \alpha \varepsilon^{X}-\gamma \varepsilon^{i}+\varepsilon^{d}\end{aligned}\end{array}$ & $\begin{array}{l}\text { Interest rate equation } \\
\qquad \begin{aligned} i & =-\frac{m-\varepsilon^{d}}{\gamma} \\
& =-\frac{\beta}{\gamma} \varepsilon^{X}-\frac{1}{\gamma} \varepsilon^{m}+\frac{1}{\gamma} \varepsilon^{d}\end{aligned}\end{array}$ \\
\hline Shock flow & Shock flow \\
\hline $\begin{aligned} i & \leftarrow \varepsilon^{X}, \varepsilon^{i} \\
m & \leftarrow \varepsilon^{X}, \varepsilon^{i}, \varepsilon^{d}\end{aligned}$ & $\begin{aligned} i & \leftarrow \varepsilon^{X}, \varepsilon^{m}, \varepsilon^{d} \\
m & \leftarrow \varepsilon^{X}, \varepsilon^{m}\end{aligned}$ \\
\hline
\end{tabular}

In contrast, in the unconventional policy regime, the central bank expands its balance sheet under the ZLB. Unconventional policies can be classified into "quantitative easing" and "credit easing," depending on whether the central bank focuses on the liability side or the asset side of its balance sheet. Quantitative easing aims to expand the liability side of the central bank's balance sheet, pumping more and more reserves into the banking system; the composition of loans and securities on the asset side of the balance sheet is incidental. The overall stance of its policy is gauged primarily in terms of its target for bank reserves. On the other hand, credit easing aims to lower longer-term market interest rates and various credit spreads, focusing on the asset side of the central bank's balance sheet, i.e., on the mix of loans and securities it holds and on how this composition of assets affects credit conditions for households and businesses. The credit easing approach - in contrast to the quantitative easing approach - is not easily summarized by a single number, such as the quantity of bank reserves or the size of the monetary base. However, credit easing resembles quantitative easing in one respect: it involves an expansion of the central bank's balance sheet. The central bank cannot operate on just one side of the balance sheet, and the only way it can buy assets (which is credit easing) is by increasing bank reserves (which is quantitative easing). To formularize a unified identification scheme, a key aspect here is that the 
implementation of unconventional policy, in both the quantitative easing and the credit easing approach, is associated with an increase in bank reserves. In the unconventional policy regime of our model, as shown in Equation (2a), the central bank controls bank reserves $(m)$ in response to macroeconomic shocks $\left(\varepsilon^{X}\right)$ associated with inflation and the output gap. A deviation from this rule is defined as a monetary policy shock $\left(\varepsilon^{m}\right)$ in the unconventional policy regime. In this regime, the short-term interest rate $(i)$ is essentially determined by the bank reserves demand function, as in Equation (2b).

With this unified identification scheme, a reserve demand shock $\left(\varepsilon^{d}\right)$ is fully accommodated by the central bank in the conventional policy regime, affecting the quantity of bank reserves $(m)$ and not the short-term interest rate $(i)$, while in the unconventional policy regime the shock $\varepsilon^{d}$ may affect the short-term interest rate $(i)$ and not bank reserves $(m)$. This regime-dependent constraint is incorporated into the TVP-VAR model as outlined below. ${ }^{4}$

\subsection{TVP-VAR model}

In the following analysis, the TVP-VAR model is formulated for the responses of the time series $Z_{t}=\left(\pi_{t}, y_{t}, i_{t}, m_{t}, \ell_{t}\right)^{\prime}$, consisting of inflation $\left(\pi_{t}\right)$, the output gap $\left(y_{t}\right)$, the short-term interest rate $\left(i_{t}\right)$, bank reserves $\left(m_{t}\right)$, and the long-term interest rate $\left(\ell_{t}\right)$. Specifically,

$$
Z_{t}=c_{t}+B_{1 t} Z_{t-1}+\cdots+B_{q t} Z_{t-q}+e_{t}, \quad e_{t} \sim N\left(0, \Sigma_{t}\right)
$$

where $c_{t}$ is a vector of time-varying intercepts, and the $B_{j t}$ are matrices of time-varying coefficients. In order to identify the structural shocks, a key modeling strategy here is to set the constraints in the time-varying variance-covariance matrix, which is decomposed as $\Sigma_{t}=A_{t}^{-1} \Lambda_{\mathrm{t}}\left(A_{t}^{-1}\right)^{\prime}$, where $\Lambda_{t}=\operatorname{diag}\left(\sigma_{\pi t}^{2}, \sigma_{y t}^{2}, \sigma_{i t}^{2}, \sigma_{m t}^{2}, \sigma_{\ell t}^{2}\right)$ is a diagonal matrix of the variances of structural shocks $\varepsilon_{t}$, and $A_{t}$ is a matrix of time-varying simultaneous relations structured as

\footnotetext{
${ }^{4}$ Iwata (2010) employs a similar approach to the regime-switching identification as this paper, but uses a constant-parameter VAR model to identify policy shocks.
} 


$$
A_{t}=\left[\begin{array}{ccccc}
1 & 0 & 0 & 0 & 0 \\
a_{\pi y, t} & 1 & 0 & 0 & 0 \\
a_{\pi i, t} & a_{y i, t} & 1 & a_{m i, t} & 0 \\
a_{\pi m, t} & a_{y m, t} & a_{i m, t} & 1 & 0 \\
a_{\pi \ell, t} & a_{y \ell, t} & a_{i \ell, t} & a_{m \ell, t} & 1
\end{array}\right]
$$

This decomposition implies

$$
e_{t}=A_{t}^{-1} \varepsilon_{t}=\left[\begin{array}{ccccc}
1 & 0 & 0 & 0 & 0 \\
\tilde{a}_{\pi y, t} & 1 & 0 & 0 & 0 \\
\tilde{a}_{\pi i, t} & \tilde{a}_{y i, t} & 1 & \tilde{a}_{m i, t} & 0 \\
\tilde{a}_{\pi m, t} & \tilde{a}_{y m, t} & \tilde{a}_{i m, t} & 1 & 0 \\
\tilde{a}_{\pi \ell, t} & \tilde{a}_{y \ell, t} & \tilde{a}_{i \ell, t} & \tilde{a}_{m \ell, t} & 1
\end{array}\right]\left[\begin{array}{c}
\varepsilon_{t}^{\pi} \\
\varepsilon_{t}^{y} \\
\varepsilon_{t}^{i} \\
\varepsilon_{t}^{m} \\
\varepsilon_{t}^{\ell}
\end{array}\right],
$$

where $\varepsilon_{t} \sim N\left(0, \Lambda_{t}\right)$. The monetary policy shock is $\varepsilon_{t}^{i}$ in the conventional policy regime and $\varepsilon_{t}^{m}$ in the unconventional policy regime. The identification scheme discussed above leads to explicit constraints: $\tilde{a}_{m i, t}=0$ in the conventional policy regime (i.e., the reserve demand shock $\varepsilon_{t}^{m}$ does not simultaneously affect the short-term interest rate $i_{t}$ ), and $\tilde{a}_{i m, t}=0$ in the unconventional policy regime (i.e., reserve demand shocks may simultaneously cause a change in the short-term interest rate $\varepsilon_{t}^{i}$, but this does not affect bank reserves $m_{t}$ ). For other parts of the simultaneous relation, the identification is based on a standard recursive restriction concerning the speed with which variables respond to shocks; that is, inflation is assumed to respond last, and financial markets (i.e., long-term interest rates) are assumed to be the most responsive. With these constraints, all the structural shocks can be just-identified, and we can interpret the restriction as a recursive identification switching between the orderings $\left(\pi_{t}, y_{t}, i_{t}, m_{t}, \ell_{t}\right)$ and $\left(\pi_{t}, y_{t}, m_{t}, i_{t}, \ell_{t}\right)$ depending on the policy regime.

The assumption of the time-varying simultaneous relations is important with respect to several possible changes through the conventional and unconventional policy regimes in the monetary policy reaction and transmission mechanisms. In the conventional policy regime, a change in the central bank's reaction function is measured by the simultaneous relations $\tilde{a}_{\pi i, t}$ and $\tilde{a}_{y i, t}$, i.e., the reaction of the short-term interest rate to inflation and output gap shocks. As the short-term interest rate approaches the ZLB, $\tilde{a}_{\pi i, t}$ and $\tilde{a}_{y i, t}$ are expected to become smaller. In the same manner, changes in the policy stance in the unconventional policy regime are measured by the parameters 
$\tilde{a}_{\pi m, t}$ and $\tilde{a}_{y m, t}$, which are expected to be close to zero in the conventional policy regime. The parameter $\tilde{a}_{m \ell, t}$ refers to the simultaneous response of the long-term yield to the structural shock associated with bank reserves. It mainly measures the portfolio rebalancing effect and/or the signaling effect of the unconventional policy, and may differ in value between quantitative easing and credit easing, reflecting differences in monetary policy actions such as the composition of asset purchases.

All the coefficients and simultaneous relations in the TVP-VAR model are assumed to follow a stationary AR(1) process:

$$
\lambda_{j k, t}=\mu_{j k}+\phi_{j k}\left(\lambda_{j k, t-1}-\mu_{j k}\right)+\eta_{j k, t}, \quad \eta_{j k, t} \sim N\left(0, v_{j k}^{2}\right),
$$

where $\left|\phi_{j k}\right|<1$. The time-varying parameters are usually modeled as following a random walk process, which aims at allowing for the possibility of permanent shifts and reducing the number of parameters, as discussed by Primiceri (2005). We instead introduce the stationary process, because a highly-persistent $\operatorname{AR}(1)$ process with $\phi_{j k}$ close to one can generate a parameter trajectory of seemingly permanent shifts in a finite sample, and because the stationarity of the latent process yields more appropriate estimates of the simultaneous relations in the latent threshold modeling introduced below (Nakajima and West 2013).

In addition, we incorporate a stochastic volatility model to describe persistent changes in the variances of the structural shocks. This is empirically crucial: it is reasonable to assume that the size of exogenous policy and non-policy shocks differs in each period, as widely discussed in the literature (see, e.g., Cogley and Sargent 2005, Primiceri 2005). In the analysis of both conventional and unconventional monetary policy regimes, monetary policy shocks associated with a regime change - such as a large change in bank reserves at the beginning and the end of the unconventional policy regime - may lead to biased parameter estimates in constant-variance VAR models. Furthermore, the stochastic volatility is adequately adapted to the case of the ZLB by reaching a sufficiently low level close to zero, if necessary. Specifically, the stochastic volatility process, by taking $h_{j, t}=\log \sigma_{j, t}^{2}$, is defined as

$$
h_{j, t}=\mu_{j}+\phi_{j}\left(h_{j, t-1}-\mu_{j}\right)+\eta_{j, t}, \quad \eta_{j, t} \sim N\left(0, v_{j}^{2}\right) \text {, }
$$

where $\left|\phi_{j}\right|<1$. 


\subsection{Latent threshold modeling}

One limitation of the standard TVP-VAR model is that the identifying restriction is fixed over time, which makes it impossible to precisely describe the regime change between the conventional and unconventional policies. To overcome this difficulty, we exploit the latent threshold model (LTM) developed by Nakajima and West (2013). The LTM can be effectively applied in cases when a parameter process such as the time-varying parameters in a VAR may be close to zero and practically insignificant in some periods. Time-varying parameter models with many practically insignificant parameters can yield increasing estimation uncertainty as the parameter dimension grows. The LTM strategy for searching for a parsimonious model structure is latent thresholding: the time-varying parameter $\lambda_{j k, t}$ is shrunk to zero when $\left|\lambda_{j k, t}\right|$ is smaller than a parameter process-specific threshold, while it maintains a non-zero time-varying value when $\left|\lambda_{j k, t}\right|$ exceeds the threshold. Data-driven inferences on thresholds and time-varying sparsity patterns induce a dynamic variable selection; i.e., the LTM structure explores the best set of variables at each time point. This approach to reducing the dimension of parameters has the great benefit of improving forecast performance and facilitating model interpretation, as shown by Nakajima and West (2013). Here, we apply and extend the LTM approach to the time-varying parameters of simultaneous relations in order to identify monetary policy shocks and to take account of the ZLB in conventional and unconventional policy regimes.

Assume an underlying latent process $\alpha_{j k, t}$, corresponding to the simultaneous relation $a_{j k, t}$, which follows the basic AR(1) process of Equation (3). The basic LTM defines

$$
a_{j k, t}=\alpha_{j k, t} s_{j k, t} \quad \text { with } \quad s_{j k, t}=I\left(\left|\alpha_{j k, t}\right| \geq d_{j k}\right)
$$

where $d_{j k}$ is the latent threshold to be estimated and $I(\cdot)$ is an indicator function that takes one when the argument holds and zero otherwise. Note that the LTM reduces to the standard $\operatorname{AR}(1)$ process when $d_{j k} \equiv 0$; therefore, a class of the LTM-based TVP-VAR models nests the standard TVP-VAR model. The underlying $\alpha_{j k, t}$ appears as a non-zero time-varying simultaneous relation in the identification matrix $A_{t}$ only when "statistically relevant" as defined by the latent threshold. Otherwise, the 
simultaneous relation is shrunk to exactly zero (Figure 1, Panel A). We introduce this LTM structure in the time-varying parameters $a_{\pi i, t}$ and $a_{y i, t}$, i.e., the simultaneous responses of the short-term interest rate to inflation and the output gap, to incorporate the effect of the ZLB, because there is little room for the central bank to lower its policy rate when the short term interest rate approaches and remains at the ZLB.

We now extend the latent threshold to a time-varying latent threshold for the regime-dependent constraint discussed above. Equation (4) is rewritten as

$$
a_{j k, t}=\alpha_{j k, t} s_{j k, t} \quad \text { with } \quad s_{j k, t}=I\left(\left|\alpha_{j k, t}\right| \geq d_{j k, t}\right),
$$

where the threshold $d_{j k, t}$ is now time dependent. If we were to assume a continuous process for $d_{j k, t}$, we would encounter an identification problem. Instead, we assume shifts of the latent threshold associated with the regimes by specifying

$$
d_{j k, t}= \begin{cases}\infty, & \text { for } t \in T \\ d_{j k}, & \text { for } t \notin T\end{cases}
$$

where $T$ is a set of time points. For the specified periods, $t \in T$, the "high-ceil" threshold, $d_{j k, t}=\infty$, always makes $a_{j k, t}$ shrink to zero (Figure 1, Panel B). For the other periods, the threshold $d_{j k}$ is a constant parameter. Modeling these two regimes enables us to estimate an overall trajectory of the time-varying parameter which is explicitly assumed to be zero for $t \in T$, but is allowed to shrink to zero for $t \notin T$.

We apply the extended LTM structure of Equations (5) and (6) to $a_{m i, t}$ and $a_{i m, t}$. For $a_{m i, t}$, we specify

$$
d_{m i, t}= \begin{cases}\infty, & \text { for } t \in T \\ d_{m i}, & \text { for } t \notin T,\end{cases}
$$

where $T$ is the conventional policy periods and $d_{m i}$ is a threshold to be estimated. With this specification, we make $a_{m i, t}$ (and hence $\tilde{a}_{m i, t}$ as the identifying restriction) explicitly shrink to zero for $t \in T$, and allow $a_{m i, t}$ to be shrunk to zero due to the ZLB for $t \notin T$, if preferred by the data. For $a_{i m, t}$, we specify

\footnotetext{
${ }^{5}$ Chan et al. (2012) develop another time-varying shrinkage method using the dynamic mixture approach, which is fundamentally related to the extended LTM structure developed here.
} 


$$
d_{i m, t}=\left\{\begin{array}{l}
\infty, \text { for } t \in T \\
0, \text { for } t \notin T
\end{array}\right.
$$

where $T$ is the unconventional policy periods. This specification means that $a_{i m, t}$ (and hence $\tilde{a}_{i m, t}$ as the identifying restriction) shrinks to zero for $t \in T$, and $a_{i m, t}$ is a time-varying parameter without the latent threshold for $t \notin T$.

To sum up, the extended LTM structure (Equations 5 and 6) is introduced in the simultaneous relations $\left(a_{m i, t}, a_{i m, t}\right)$ to embed the identifying restriction for the conventional and unconventional policy regimes, and the basic LTM (Equation 4) is applied to the simultaneous responses of the short-term interest rate to inflation and the output gap $\left(a_{\pi i, t}, a_{y i, t}\right)$ to incorporate the ZLB. Note that the extended LTM for $a_{m i, t}$ and $a_{i m, t}$ makes the model just-identified, and the basic LTM for $a_{\pi i, t}$ and $a_{y i, t}$ further examines for overidentified structures in $A_{t}$. Given the structure just-identified by $a_{m i, t}$ and $a_{i m, t}$ for all time points, it is reasonable to expect overidentification, by assuming $a_{\pi i, t}$ and/or $a_{y i, t}$ equal to zero when the short-term interest is close to zero. Assuming the possibility of overidentification, the LTM searches for the best (time-varying) set of simultaneous relations in explaining the time series responses. This is a new and efficient estimation method for exploring time-varying identification structures. In particular, it provides further insights into the effects of the ZLB by assessing when each of the parameters in the policy reaction function approaches to zero.

In order to fit the latent threshold TVP-VAR model we use Bayesian Markov chain Monte Carlo (MCMC) computational methods. Specifying prior distributions for the model hyperparameters, we sample the posterior distribution of the hyperparameters including the latent thresholds and all the state variables, given the data. Details of the specific algorithm for the TVP-VAR model can be found in Primiceri (2005) and Nakajima (2011), while the algorithm for the LTM can be found in Nakajima and West (2013). ${ }^{6}$

\footnotetext{
${ }^{6}$ It is worth mentioning that sampling the state variable in the LTM, i.e., $\alpha_{j k, t}$ in equation (5), is straightforward even with the time-varying (regime-shift) latent threshold. A sampler sequentially generates $\alpha_{j k, t}$ given the data and other parameters including the states $\left\{\alpha_{j k, \tau}, \tau \neq t\right\}$ through each $t$ with a direct Metropolis-within-Gibbs sampling strategy. This single-move sampler does not require sequential filtering or smoothing, which can be easily applied to the time-varying latent threshold (see Appendix A in Nakajima and West 2013).
} 


\section{Data}

We illustrate the approach just described by applying it to monetary policy and macroeconomic developments in Japan. The analysis uses quarterly data from 1981/Q2 to $2012 / \mathrm{Q} 3$ on the CPI inflation rate $\left(\pi_{t}\right)$, the GDP gap $\left(y_{t}\right)$, the overnight call rate $\left(i_{t}\right)$, the outstanding balance of current accounts $\left(m_{t}\right)$ held at the Bank of Japan, and the yield on 10-year Japanese government bonds $\left(\ell_{t}\right){ }^{7}$ Developments in these variables during the observation period are depicted in Figure 2.

We define the unconventional policy regime as the periods from 2001/Q1 to 2006/Q1 (labeled as UC1), and from 2010/Q1 to 2012/Q3, i.e., the end of the observation period (labeled as UC2), and the conventional policy regime as other periods, when the overnight call rate is the main operating target. ${ }^{8}$ Regarding UC1, the Bank of Japan (BOJ) introduced the quantitative easing policy (QEP) in March 2001, changing its main operating target for money market operations from the overnight call rate to the outstanding balance of current accounts held at the BOJ, and terminated QEP in March 2006. Regarding UC2, in response to the global financial crisis, the BOJ introduced a new funds-supplying operation (a fixed-rate funds-supplying operation against pooled collateral) in December 2009 in order to encourage a further decline in longer-term interest rates in the money market through provision of ample longer-term funds at an extremely low interest rate. Then, in October 2010, the BOJ introduced its policy of "comprehensive monetary easing" to further enhance monetary easing by encouraging the decline in longer-term interest rates and various risk premiums. As part of this comprehensive monetary easing policy, the BOJ established the Asset Purchase Program, a program on the BOJ's balance sheet to purchase various financial assets such as Japanese government bonds (JGBs), treasury discount bills, CP, corporate bonds,

\footnotetext{
${ }^{7}$ The CPI inflation rate here is based on the index excluding fresh food and is adjusted to remove the effect of the increase in the consumption tax. The GDP gap is calculated by the Bank of Japan (BOJ). The logarithm of the outstanding balance of current accounts (including deposits by the Japan Post and the Japan Post Bank) is used for the estimation. The sample period starts from 1981/Q2 due to the data availability of the outstanding balance of current accounts.

${ }^{8}$ In the conventional monetary policy regime, the BOJ lowered the targeted overnight call rate from $50 \mathrm{bp}$ to $25 \mathrm{bp}$ in September 1998, and implemented the zero interest rate policy from February 1999 to August 2000. Then, after the termination of its quantitative easing policy (QEP), the BOJ raised the target overnight call rate from 2006 through 2007 to 50bp, while after the global financial crisis, it lowered the target overnight call rate to as little as $10 \mathrm{bp}$ in 2008.
} 
ETFs, and Japan real estate investment trusts (J-REITs). ${ }^{9}$ As seen in Figure 2, the outstanding amount of current account balances held at the BOJ (i.e., bank reserves) significantly increased in both $\mathrm{UC} 1$ and $\mathrm{UC} 2 .^{10}$

\section{Empirical results}

For model fitting, the number of lags is set to three based on preliminary experiments. The MCMC used 50,000 iterations after a burn-in period of 10,000 samples. ${ }^{11}$ In the following analysis, posterior estimates are reported in terms of the posterior medians and $68 \%$ credible intervals.

\subsection{Simultaneous relations and identification}

Figure 3 shows summary results for the identification of the conventional and unconventional monetary policy shocks. The panels display the posterior estimates of

\footnotetext{
9 As for measuring unconventional monetary policy shocks, earlier studies often use the monetary base, which is the sum of currency in circulation and the outstanding balance of current accounts. The reason why we use the current account balances instead of the monetary base is that during our sample period the BOJ had purchased Japanese government bonds (JGBs) under two different frameworks, namely (1) for the purpose of supplying currency in line with underlying long-term developments in the economy, and (2) as outright purchases under the Asset Purchase Program (introduced as an unconventional policy measure). From the viewpoint of the Bank's balance sheet, it is appropriate that the Bank holds JGBs (long-term assets of the Bank) in accordance with the amount outstanding of banknotes in circulation (liabilities of the Bank), which increases in line with economic growth in the long run. Thus, the outright purchases of JGBs to supply currency consistent with underlying long-term developments in the economy were regularly conducted in both a conventional and an unconventional policy regime, so that it is not necessarily appropriate to use the monetary base (including the currency in circulation) as a measure of unconventional policy. In contrast, the outstanding balance of current accounts held at the Bank is closely related with the amount of outright purchases through the Asset Purchase Program and therefore more appropriate as a measure of unconventional policy.

${ }^{10}$ The current account balances increased in 2009 before UC2. However, in 2009, the BOJ conducted money market operations with the overnight call rate as the operating target, and the increase in the current account balances was not caused by a monetary policy shock but precautionary demand for reserves. Many financial institutions tried to secure more funds in response to the elevated tensions in the money market after the failure of Lehman Brothers in mid-September 2008, and this increase in reserve demand was fully accommodated by the ample provision of funds by the BOJ.

11 In the empirical analysis, the following priors are used: $\left(\phi_{j k}+1\right) / 2 \sim B(1,1),\left(\phi_{j}+\right.$ 1) $/ 2 \sim B(1,1), \mu_{j k} \sim N(0,1)$; $\exp \left(\mu_{j}\right) \sim G(15,0.03)$ for $(\pi, y), G(3,15)$ for $(i, m)$, and $G(3,3)$ for $b ; 1 / v_{j k}^{2} \sim G(20,0.001)$ for the lagged coefficients $\left(B_{i t}\right)_{j k}, G(20,0.01)$ for the simultaneous relations $a_{j k, t}$ and $\alpha_{j k, t}$; regarding the time-varying intercept, $\mu_{j k}=0, \phi_{j k}=1$, and $1 / v_{j k}^{2} \sim G(25,0.01)$ for $(\pi, i), G(50,0.01)$ for $(y, m, \ell) ; 1 / v_{j k}^{2} \sim G(10,0.01)$ for $(\pi, y)$, and $G(10,0.01)$ for $(i, m, \ell)$. For the latent threshold, $d_{j k} \sim U\left(0,\left|\mu_{j k}\right|+K u_{j k}^{1 / 2}\right)$, where $u_{j k}=v_{j k}^{2} /\left(1-\phi_{j k}^{2}\right)$, and $K=3$, following Nakajima and West (2013).
} 
the simultaneous relations associated with the structural shocks. Note that these are estimates of $A_{t}^{-1}$, not $A_{t}$. The conventional and unconventional monetary policy shocks are identified based on the regime-switching constraint, as seen in Panels (6) and (7). Based on the LTM, the time-varying parameters $\left(\tilde{a}_{m i, t}, \tilde{a}_{i m, t}\right)$ are shrunk to zero during the constraint periods, which makes the model just-identified at each time point. Panel (6) plots the trajectory of the simultaneous response of bank reserves to short-term interest rate shocks in the conventional policy regime. It shows that short-term interest rates have a negative impact on bank reserves from the late 1990s onward, which probably reflects the increase in the interest elasticity of demand for excess reserves due to the decline in the opportunity cost of holding them. In Panel (7), posterior medians are totally zero in the simultaneous response of the short-term interest rate to a bank reserve shock in the unconventional policy regime. This result is quite plausible because short-term interest rates remained at a low level while the outstanding balances of current accounts increased dramatically.

Next, Panels (2) and (3) show a marked shrinkage in the simultaneous responses of short-term interest rates to inflation and the output gap $\left(\tilde{a}_{\pi i, t}, \tilde{a}_{y i, t}\right)$ as short-term interest rates approached zero in the 1990s. This shrinkage is induced by the LTM, indicating that the ZLB constraint has been relevant since the early 2000s and the over-identifying restrictions are valid. However, these time-varying parameters temporarily become somewhat positive after the termination of QEP, when the BOJ raised the target overnight call rate from 2006 through 2007, which implies that the just-identifying restrictions are valid during this period.

Panels (4) and (5) show the dynamics of the simultaneous responses of bank reserves to inflation and the output gap $\left(\tilde{a}_{\pi m, t}, \tilde{a}_{y m, t}\right)$. These parameters are estimated to be around zero in the conventional policy regime, but are substantially negative in the unconventional policy regime, implying that the BOJ increases bank reserves in response to negative inflation and output gap shocks.

Another important result is the simultaneous response of long-term interest rates to a bank reserve shock $\left(\tilde{a}_{m \ell, t}\right)$, which is plotted in Panel (11). The parameter is clearly negative during the unconventional policy regime, which implies that increasing 
bank reserves lowers long-term interest rates, consistent with the portfolio rebalancing effect and/or signaling effect of unconventional policy. Furthermore, the trajectory shows that a bank reserve shock has a larger impact on long-term interest rates in UC2 than UC1, which possibly reflects differences in the way bank reserves were expanded. In the QEP during UC1, the BOJ aimed to expand the liability side of its balance sheet by mainly increasing the outright purchase of long-term JGBs as well as conducting the short-term funds-supplying operations. In UC2, in contrast, the BOJ has purchased various financial assets, including riskier market products $(\mathrm{CP}$, corporate bonds, ETFs, and J-REITs) as well as JGBs, which may have resulted in a larger impact of the expansion of bank reserves on financial markets.

The important role of the LTM in the TVP-VAR model can be seen by comparing the results in Figure 3 with those in Figure 4, which is based on the model without the latent thresholds for the ZLB. A criticism in the TVP-VAR context is that the simultaneous responses of short-term interest rates to inflation and the output gap $\left(\tilde{a}_{\pi i, t}, \tilde{a}_{y i, t}\right)$ are often estimated to be non-zero values, even when the ZLB is binding. ${ }^{12}$ This problem can be seen in Figure 4 (2)(3), while it is clearly resolved with the LTM, as shown in Figure 3 (2)(3). In addition, the credible intervals for several time-varying parameters, including parameters other than the ZLB-related ones, are wider in the model without latent thresholds than those with latent thresholds for the ZLB, as can be seen by comparing Figures 3 and 4. The credible intervals of the simultaneous response of long-term interest rates to bank reserve shocks $\left(\tilde{a}_{m \ell, t}\right)$ do include zero in the model without latent thresholds (Figure 4 (11)), while those in the model with latent thresholds (Figure 3 (11)) do not include zero during the unconventional policy regime. Because the LTM-based TVP-VAR models nests the standard TVP-VAR model, the significant shrinkage of the parameters $\left(\tilde{a}_{\pi i, t}, \tilde{a}_{y i, t}\right)$ for the ZLB observed in Figure 3 indicates that the LTM-based time-varying parameters are preferred by the data. This confirms that shrinking time-varying parameters clearly results in a properly defined and parsimonious model, thus confirming the advantage of the LTM strategy.

$\overline{12}$ See, for example, Nakajima (2011) for more on this problem. 


\subsection{Volatility of identified structural shocks}

Figure 5 exhibits the trajectories of the stochastic volatility of the identified shocks, $\sigma_{j t}=\exp \left(h_{j t} / 2\right)$. The estimates of inflation and output gap shocks show high volatility in the second half of the 2000s due to the impact of the rise in global commodity prices on inflation and due to the impact of the global financial crisis on output gap. In addition, the rise in the volatility of output gap shocks possibly resulted from a decline in the natural rate of interest rate due to rapid population aging and Japan's delayed response to globalization. ${ }^{13}$

Regarding monetary policy measures, the volatility of short-term interest rates has remained at a much lower level since the mid-1990s than before in the presence of the ZLB. The volatility of bank reserves is clearly time-varying: the estimates show periods of high-volatility related to the Y2K problem (around the year 2000), to the increase in the target for current account balances in UC1 (2001-2003), to the termination of UC1 (in 2006), and to the start of UC2 (since 2010). The volatility of long-term interest rates appears to have followed a moderate downward trend since the 1990s as their level gradually declined.

As discussed in the literature (e.g., Cogley and Sargent 2005, Primiceri 2005), these stochastic volatilities play an important role in accounting for changes in the size of structural shocks regarding both monetary policy measures and other macroeconomic variables when trying to identify monetary policy shocks.

\subsection{Impulse response analysis}

Figure 6 explores the monetary policy reaction functions by plotting the impulse responses of short-term interest rates and bank reserves to inflation and output gap shocks. The trajectories show the impact one year after the shocks. In the 1980s, the reaction function resembles the Taylor rule (with an inflation coefficient of around 1.5 and an output gap coefficient of around 0.5). In the 1990s, however, the impulse responses declined as the ZLB is binding. The estimation results also indicate that in the unconventional policy regime bank reserves clearly respond to inflation and output gap shocks.

13 See Shirakawa (2011) for details. 
Figure 7 displays the impulse responses of inflation and the output gap to monetary policy shocks (two years after the shock in the case of the former and one year after in the case of the latter). ${ }^{14}$ For the conventional monetary policy regime, the estimates imply that the impact of a short-term interest rate shock on inflation and the output gap was relevant in the 1980s, but that it gradually declined after the burst of the bubble economy at the beginning of the 1990s. ${ }^{15}$ Figure 8 shows the impulse responses of inflation and the output gap to a negative long-term interest rate shock, which also weakened after the bubble burst. ${ }^{16}$ The interest rate elasticity of private expenditures may have declined when the Japanese economy was struggling with the deleveraging problem following the burst of the bubble. In addition, as shown in Figure 9, the response of inflation to an output gap shock shows a moderate decline since the 1990s, which implies a flatter slope of the Philips curve and is consistent with many previous studies. ${ }^{17}$ These developments result in the lowering impact of monetary easing on the real economy and inflation in the 1990s.

For the unconventional monetary policy regime, the impact of a bank reserve shock on inflation and the output gap is positive but very uncertain, as shown in Figure 7. Although the impact of a bank reserve shock on long-term interest rates is relevant in the unconventional policy regime (see Figure $3(11)$ ), the credible intervals for the responses of inflation and the output gap to a negative long-term interest rate shock become wider from the late 1990s (see Figure 8). The long-term interest rate could not change as much as before because it had reached a very low level, and the volatilities of inflation and output gap shocks are clearly large (see Figure 5), both of which probably make the credible intervals for the impulse responses of inflation and the output gap

\footnotetext{
${ }^{14}$ Our estimation results (omitted here) show that the length of the transmission lag from a monetary policy shock to inflation is longer than that to the output gap.

${ }^{15}$ Since the mid-1990s, the structural shock of short-term interest rates diminished due to the ZLB, which leads to insignificant impulse responses of inflation and the output gap.

${ }^{16}$ We can regard this structural shock mainly as a term premium shock, because we specify the recursive identification between short-term and long-term interest rates: the structural shock associated with long-term interest rates excludes the effect caused by a change in the short-term interest rate.

17 The increase in the degree of nominal rigidity (e.g., a decline in the frequency of price adjustment due to the decline in the inflation rate) and real rigidity (e.g., a rise in the price elasticity of demand due to the increased competition) leads to flattening of the Philips curve. See, for example, De Veirman (2009) for evidence of a flatter Phillips curve in Japan.
} 
wider. In principle, the TVP-VAR is a multivariate regression model; that is, relatively smaller variances in the independent variable and larger volatility in the residuals generally yield larger uncertainty in the regression coefficients. This leads to the greater uncertainty in the impulse response analysis shown in Figure 8. Overall, the empirical evidence suggests that the impact of the unconventional monetary policy on the financial market is relevant, but the transmission effect from the financial market on the real economy involve considerable uncertainty, which leads to the wide credible intervals for the impact of unconventional policy actions on inflation and the output gap.

\section{Concluding remarks}

This paper proposed a new estimation framework for identifying monetary policy shocks in both conventional and unconventional policy regimes, exploiting TVP-VAR and latent threshold model (LTM) techniques. We incorporated a regime-switching identification and a time-varying overidentification for the ZLB using the LTM. The empirical analysis of Japan's monetary policy illustrates the usefulness of the proposed approach for the detection of the conventional and unconventional monetary policy shocks. The estimation results suggest that the impact of unconventional monetary policy shocks on the real economy and inflation was positive but very uncertain, partly because the volatility of inflation and output gap shocks has increased after the financial crisis and this makes it difficult to quantify precisely the transmission effects of changes in financial conditions on the real economy and inflation.

An advantage of the proposed approach is that we can employ TVP-VAR analysis to both conventional and unconventional policy regimes without dividing the observation period into subperiods. Japan was the front-runner of the unconventional policy regime, embarking on a policy of quantitative easing in the early 2000s. Since then, the BOJ has implemented the unconventional policy actions for considerable years, and the adequate time series data in both the conventional and unconventional policy regimes enable us to provide the empirical analysis in this paper. After the global financial crisis, central banks such as Federal Reserve and Bank of England have implemented the unconventional policy actions. Measuring their effect on the economy 
using our estimation framework, after more data periods are available, is of interest, which remains as a future work.

We note the following limitation in our approach. As often discussed in the literature, there are three main transmission channels through which unconventional monetary policy actions affect asset prices: the policy signaling channel (i.e., unconventional monetary policy actions affect asset prices through news on expected future policy interest rates), the portfolio rebalancing channel (i.e., policy actions result in a rebalancing of investors' portfolios due to imperfect asset substitutability), and the liquidity premium channel (i.e., policies affect asset prices by improving market function and reducing premiums for illiquidity). ${ }^{18}$ Technically, focusing on the unconventional monetary policy shocks associated with changes in bank reserves, the current model does not distinguish among the three transmission channels, which may affect financial markets differently. Furthermore, the effect of forward guidance regarding the future path of interest rates is an important aspect of monetary policy in circumstances where the ZLB plays a role. This effect is included in the long-term interest rate shock in the current specification; however, it is impossible in the current setting to identify shocks associated with forward guidance as monetary policy shocks, because long-term interest rate shocks also include changes in term premiums (caused by factors other than the portfolio rebalancing effect of the central bank's asset purchases). ${ }^{19}$ In order to identify the shocks associated with forward guidance, incorporating yield curve information into the framework proposed here is a potentially instructive extension that is left for the future.

\footnotetext{
${ }^{18}$ See, for example, Joyce et al. (2012) for a discussion of the transmission mechanisms through which central bank asset purchases affect asset prices.

${ }^{19}$ For example, the government's debt management policy may affect the term premiums of the long-term interest rate.
} 


\section{References}

Bauer, M. D., and G. D. Rudebusch (2011), "The Signaling Channel for Federal Reserve Bond Purchases," Working Paper Series, No. 2011-21, Federal Reserve Bank of San Francisco.

Baumeister, C., and L. Benati (2010), "Unconventional Monetary Policy and the Great Recession - Estimating the Impact of a Compression in the Yield Spread at the Zero Lower Bound," Working Paper Series, No. 1258, European Central Bank.

Chan, J. C., G. Koop, R. Leon-Gonzalez, and R. W. Strachan (2012), "Time Varying Dimension Models," Journal of Business and Economic Statistics, 30(3), pp. 358-367.

Chen, H., V. Cúrdia, and A. Ferrero (2012), "The Macroeconomic Effects of Large-Scale Asset Purchase Programs," Economic Journal, 122, pp. F289-315.

Chung, H., J. P. Laforte, D. Reifschneider, and J. C. Williams (2012), "Have We Underestimated the Likelihood and Severity of Zero Lower Bound Events?" Journal of Money, Credit and Banking, 44(s1), pp. 47-82.

Cogley, T., and T. J. Sargent (2005), "Drifts and Volatilities: Monetary Policies and Outcomes in the Post WWII U.S.," Review of Economic Dynamics, 8(2), pp. 262-302.

De Veirman, E. (2009), "What Makes the Output-Inflation Trade-Off Change?” Journal of Money, Credit and Banking, 41(6), pp. 1117-1140.

Fuhrer, J. C., and G. P. Olivei (2011), "The Estimated Macroeconomic Effects of the Federal Reserve's Large-Scale Treasury Purchase Program," Public Policy Briefs, No. 2011-2, Federal Reserve Bank of Boston.

Gagnon, J. E., M. Raskin, J. Remache, and B. Sack (2011), "The Financial Market Effects of the Federal Reserve's Large-Scale Asset Purchases," International Journal of Central Banking, 7(1), pp. 3-43.

Hamilton, J. D., and J. C. Wu (2012), "The Effectiveness of Alternative Monetary Policy Tools in a Zero Lower Bound Environment," Journal of Money, Credit and Banking, 44(s1), pp. 3-46.

Iwata, S. (2010), "Monetary Policy and the Term Structure of Interest Rates When Short-Term Rates Are Close to Zero," Monetary and Economic Studies, 28, pp. 59-77.

Joyce, M., A. Lasaosa, I. Stevens, and M. Tong (2011), "The Financial Market Impact of Quantitative Easing in the United Kingdom," International Journal of Central Banking, 7(3), pp. 113-161. 
Joyce, M., D. Miles, A. Scott, and D. Vayanos (2012), "Quantitative Easing and Unconventional Monetary Policy - An Introduction," Economic Journal, 122, pp. F271-288.

Kapetanios, G., H. Mumtaz, I. Stevens, and K. Theodoridis (2012), "Assessing the Economy-Wide Effects of Quantitative Easing,” Economic Journal, 122, pp. F316-347.

Kiley, M. (2012), "The Aggregate Demand Effects of Short- and Long-Term Interest Rates," Finance and Economics Discussion Series, No. 2012-54, Federal Reserve Board.

Kimura, T., and D. H. Small (2006), "Quantitative Monetary Easing and Risk in Financial Asset Markets," Topics in Macroeconomics, 6(1), pp. 1-54.

Nakajima, J. (2011), "Time-Varying Parameter VAR Model with Stochastic Volatility: An Overview of Methodology and Empirical Application," Monetary and Economic Studies, 29, pp. 81-103.

Nakajima, J., and M. West (2013), "Bayesian Analysis of Latent Threshold Dynamic Models," Journal of Business and Economic Statistics, 31(2), in press.

Primiceri, G. E. (2005), “Time Varying Structural Vector Autoregressions and Monetary Policy," Review of Economic Studies, 72(3), pp. 821-852.

Shirakawa, M. (2011), "Globalization and Population Aging: Challenges Facing Japan," Speech to the Board of Councillors of Nippon Keidanren (Japan Business Federation) in Tokyo, Bank of Japan, December 22, 2011.

Stein, J. C. (2012), "Evaluating Large-Scale Asset Purchases," Remarks, Federal Reserve Board, October 11, 2012.

Ueda, K. (2012), "The Effectiveness of Non-Traditional Monetary Policy Measures: The Case of the Bank of Japan,” Japanese Economic Review, 63(1), pp. 1-22.

Wright, J. H. (2012), "What does Monetary Policy do to Long-term Interest Rates at the Zero Lower Bound?” Economic Journal, 122, pp. F447-466. 
Figure 1. Conceptual representation of latent threshold models (LTMs)

(A) Basic LTM
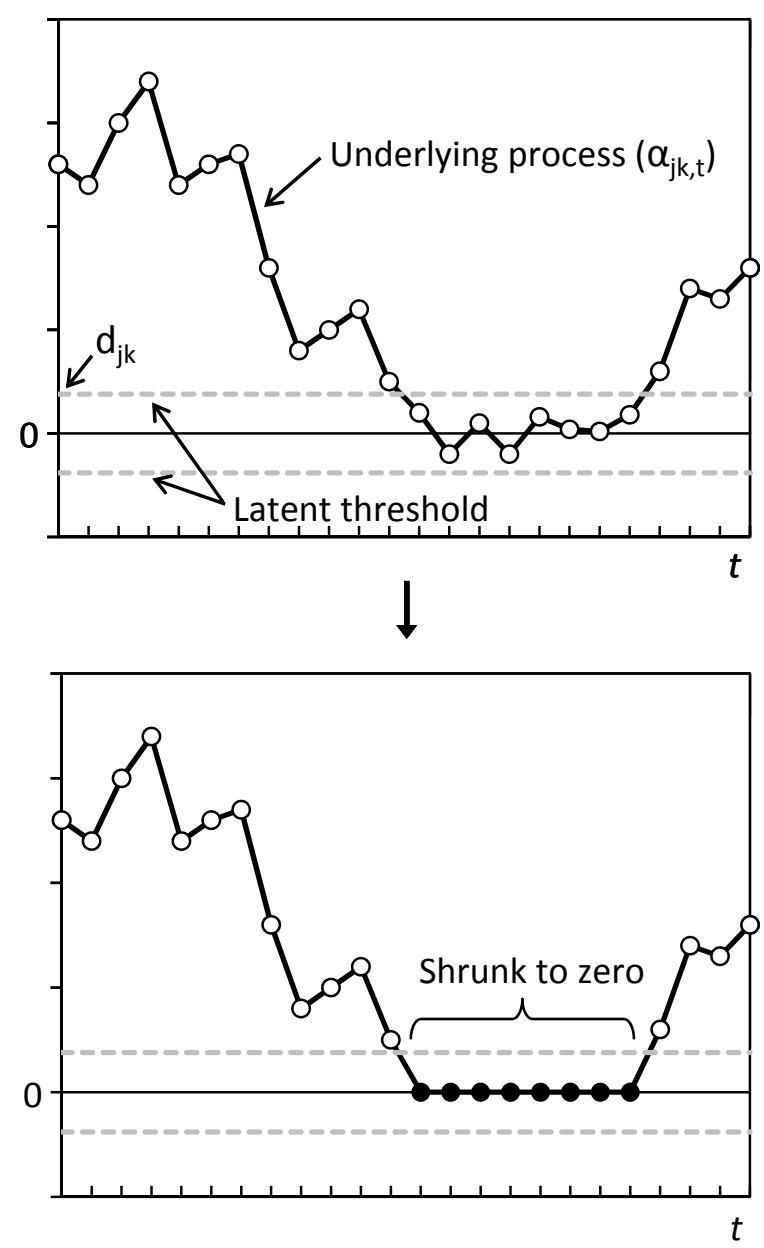

(B) LTM with time-varying latent threshold
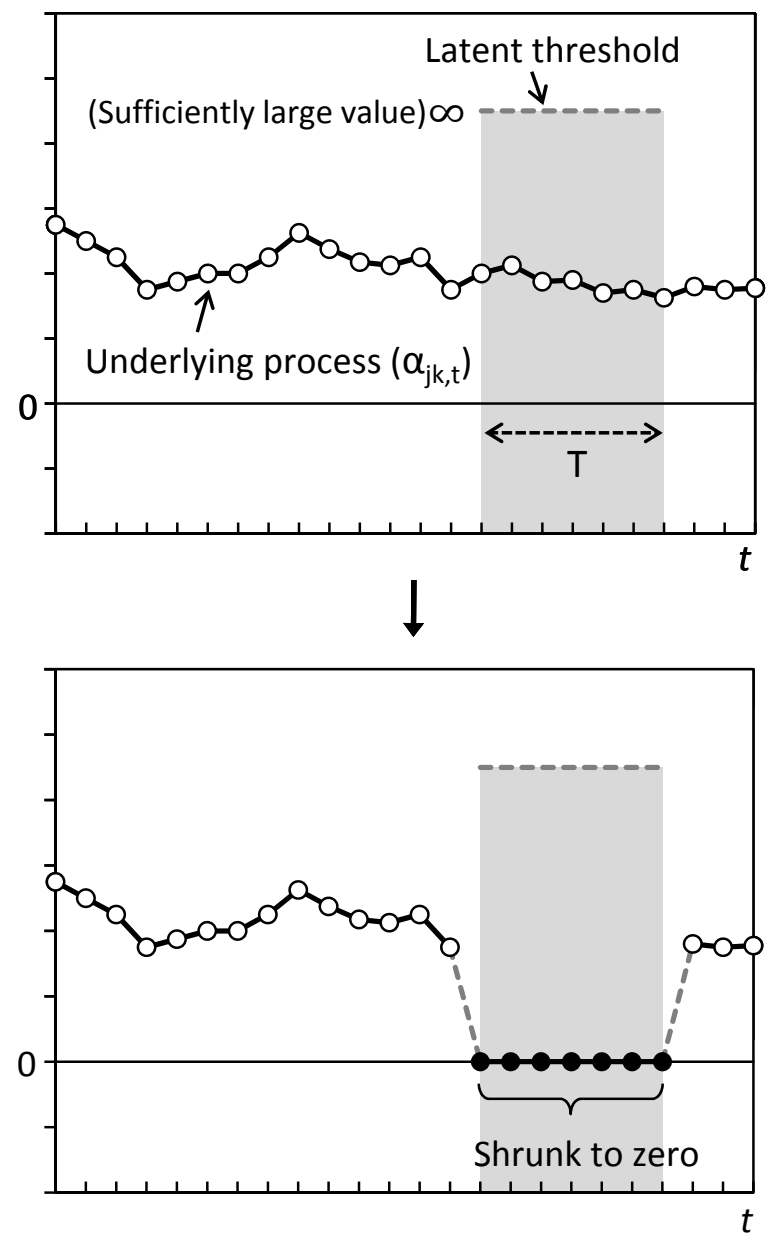
Figure 2. Japanese macroeconomic data
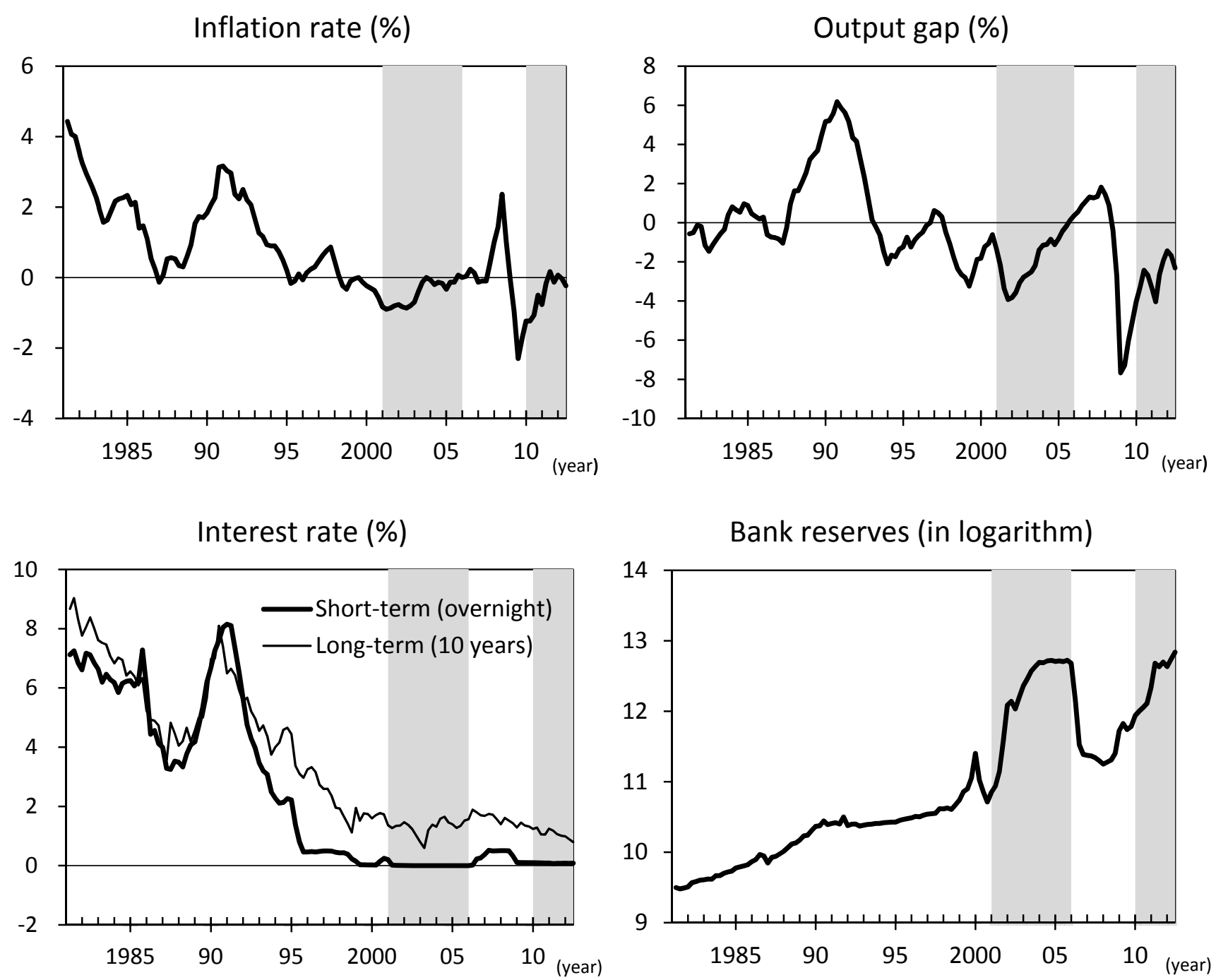

Note: Shadowed periods represent unconventional policy regime periods in the estimated model. 
Figure 3. Posterior estimates of simultaneous relations with latent thresholds

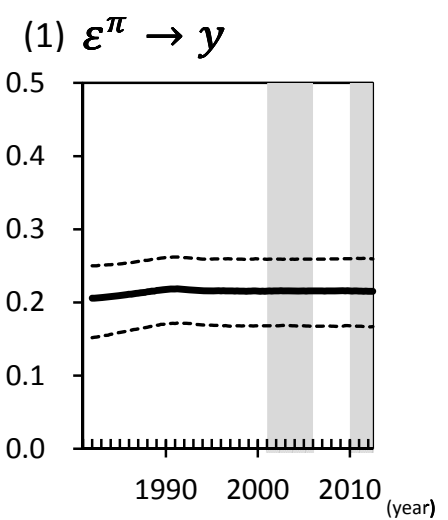

$$
\left[\begin{array}{c}
Y_{t}^{\pi} \\
Y_{t}^{y} \\
Y_{t}^{i} \\
Y_{t}^{m} \\
Y_{t}^{\ell}
\end{array}\right]=\left[\begin{array}{ccccc}
1 & 0 & 0 & 0 & 0 \\
\tilde{a}_{\pi y, t} & 1 & 0 & 0 & 0 \\
\tilde{a}_{\pi i, t} & \tilde{a}_{y i, t} & 1 & \tilde{a}_{m i, t} & 0 \\
\tilde{a}_{\pi m, t} & \tilde{a}_{y m, t} & \tilde{a}_{i m, t} & 1 & 0 \\
\tilde{a}_{\pi \ell, t} & \tilde{a}_{y \ell, t} & \tilde{a}_{i \ell, t} & \tilde{a}_{m \ell, t} & 1
\end{array}\right]\left[\begin{array}{c}
\varepsilon_{t}^{\pi} \\
\varepsilon_{t}^{y} \\
\varepsilon_{t}^{i} \\
\varepsilon_{t}^{m} \\
\varepsilon_{t}^{\ell}
\end{array}\right]
$$

$\pi$ : inflation rate, $y$ : output gap, $i$ : short-term interest rate, $m$ : bank reserves, $\ell$ : long-term interest rate

$\begin{array}{ll}\text { (2) } \varepsilon^{\pi} \rightarrow i & \text { (3) } \varepsilon^{y} \rightarrow i\end{array}$

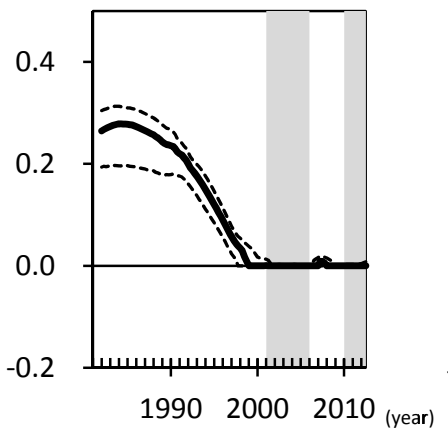

(4) $\varepsilon^{\pi} \rightarrow m$

0.2

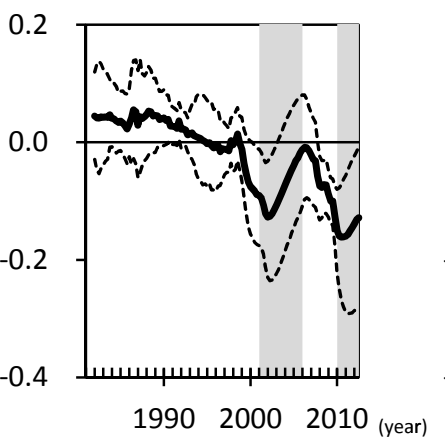

(8) $\varepsilon^{\pi} \rightarrow \ell$

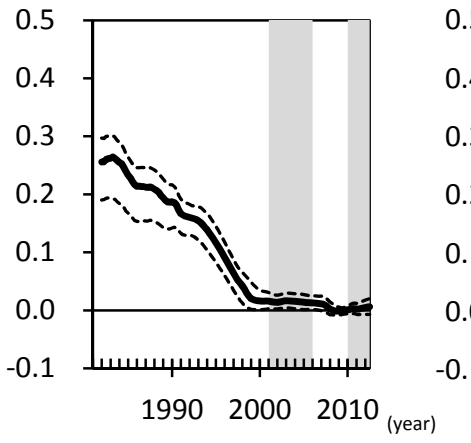

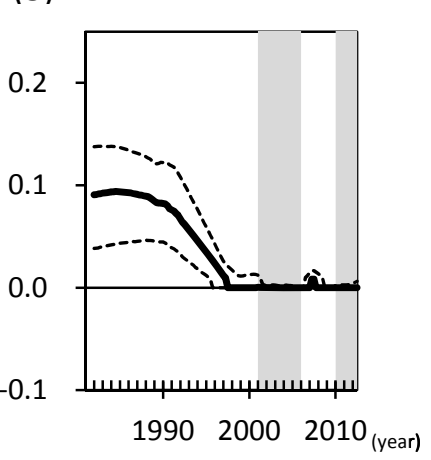

(5) $\varepsilon^{y} \rightarrow m$

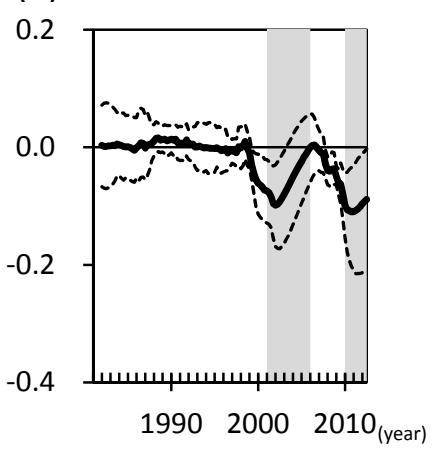

(6) $\varepsilon^{i} \rightarrow m$

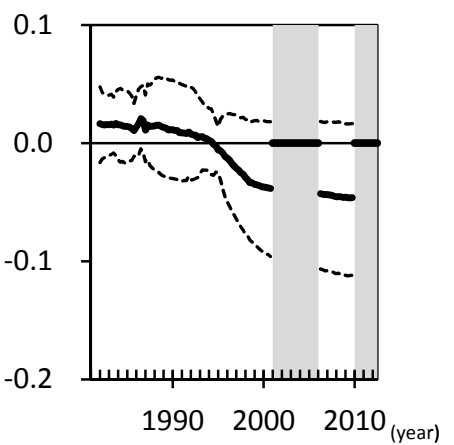

(10) $\varepsilon^{i} \rightarrow \ell$

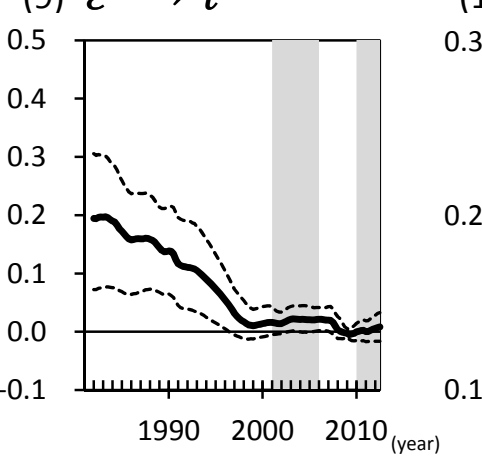

0.3
0.2

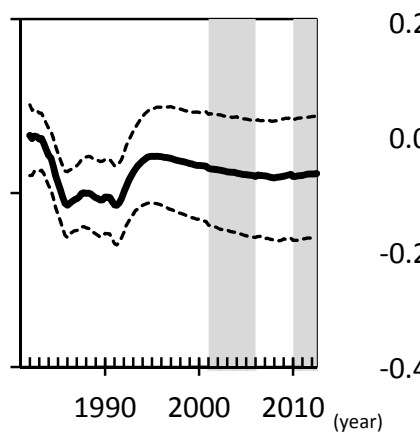

(11) $\varepsilon^{m} \rightarrow \ell$

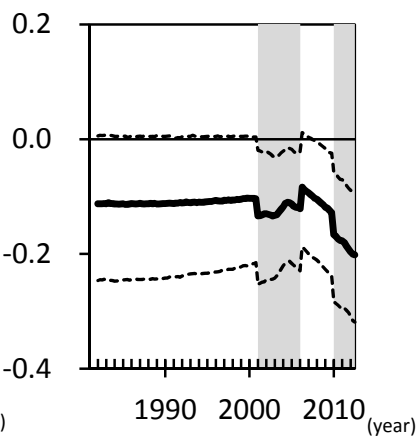

Note: Posterior medians with $68 \%$ credible intervals. 
Figure 4. Posterior estimates of simultaneous relations without ZLB latent thresholds

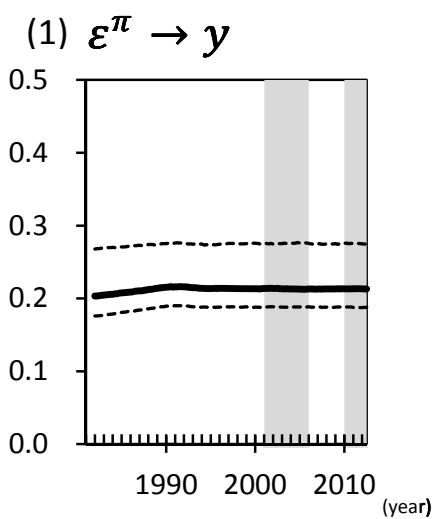

$$
\left[\begin{array}{c}
Y_{t}^{\pi} \\
Y_{t}^{y} \\
Y_{t}^{i} \\
Y_{t}^{m} \\
Y_{t}^{\ell}
\end{array}\right]=\left[\begin{array}{ccccc}
1 & 0 & 0 & 0 & 0 \\
\tilde{a}_{\pi y, t} & 1 & 0 & 0 & 0 \\
\tilde{a}_{\pi i, t} & \tilde{a}_{y i, t} & 1 & \tilde{a}_{m i, t} & 0 \\
\tilde{a}_{\pi m, t} & \tilde{a}_{y m, t} & \tilde{a}_{i m, t} & 1 & 0 \\
\tilde{a}_{\pi \ell, t} & \tilde{a}_{y \ell, t} & \tilde{a}_{i \ell, t} & \tilde{a}_{m \ell, t} & 1
\end{array}\right]\left[\begin{array}{c}
\varepsilon_{t}^{\pi} \\
\varepsilon_{t}^{y} \\
\varepsilon_{t}^{i} \\
\varepsilon_{t}^{m} \\
\varepsilon_{t}^{\ell}
\end{array}\right]
$$

$\pi$ : inflation rate, $y$ : output gap, $i$ : short-term interest rate, $m$ : bank reserves, $\ell$ : long-term interest rate

(2) $\varepsilon^{\pi} \rightarrow i$

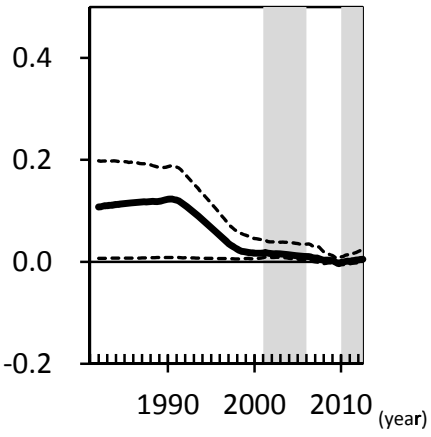

(4) $\varepsilon^{\pi} \rightarrow m$

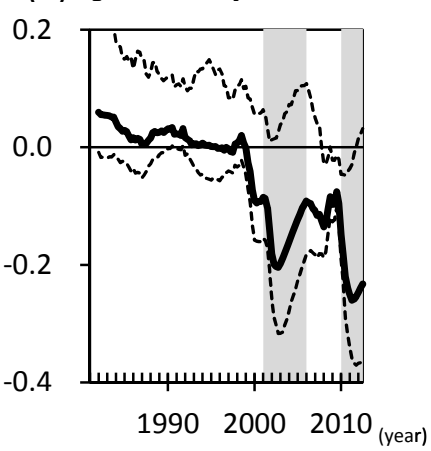

(8) $\varepsilon^{\pi} \rightarrow \ell$

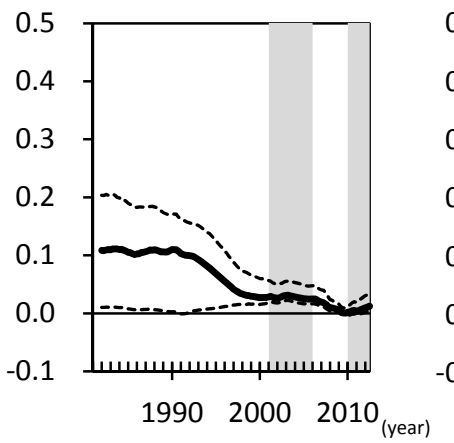

(3) $\varepsilon^{y} \rightarrow i$

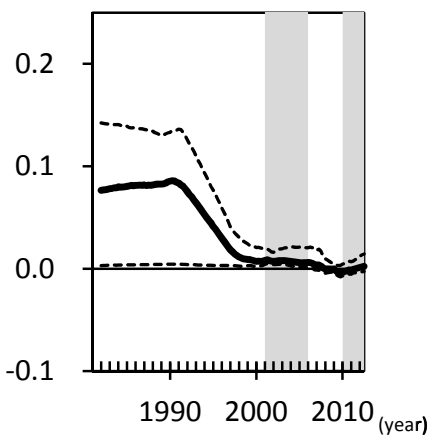

(5) $\varepsilon^{y} \rightarrow m$

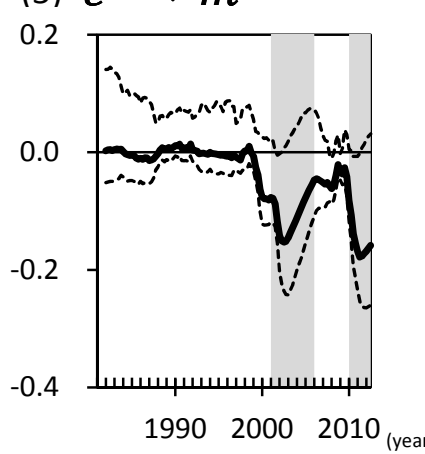

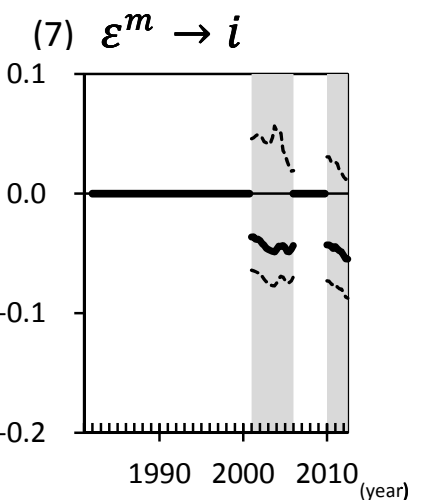

(6) $\varepsilon^{i} \rightarrow m$

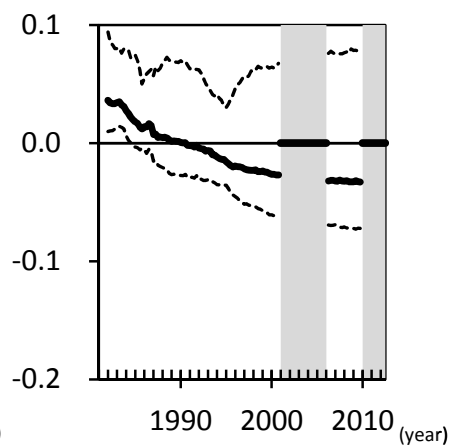

(9) $\varepsilon^{y} \rightarrow \ell$

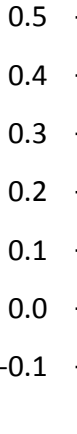

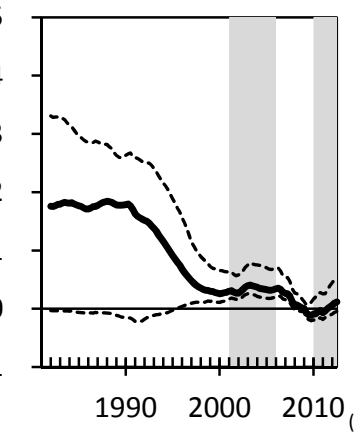

(10) $\varepsilon^{i} \rightarrow \ell$

0.4

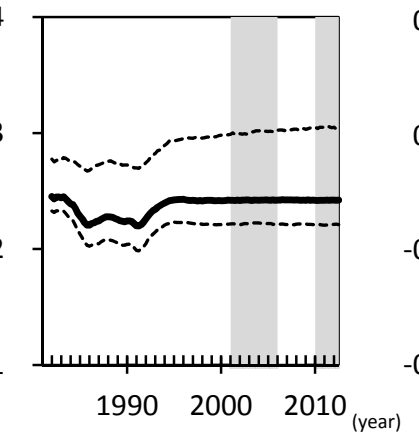

(11) $\varepsilon^{m} \rightarrow \ell$

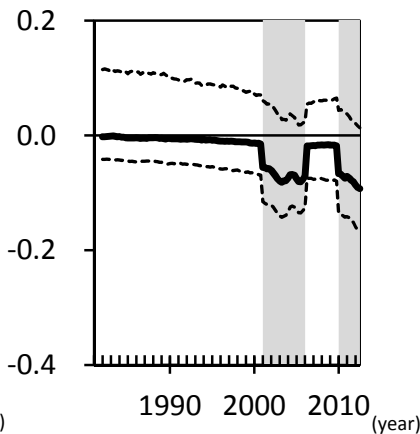

Note: Posterior medians with $68 \%$ credible intervals. 
Figure 5. Posterior estimates of stochastic volatility for structural shocks
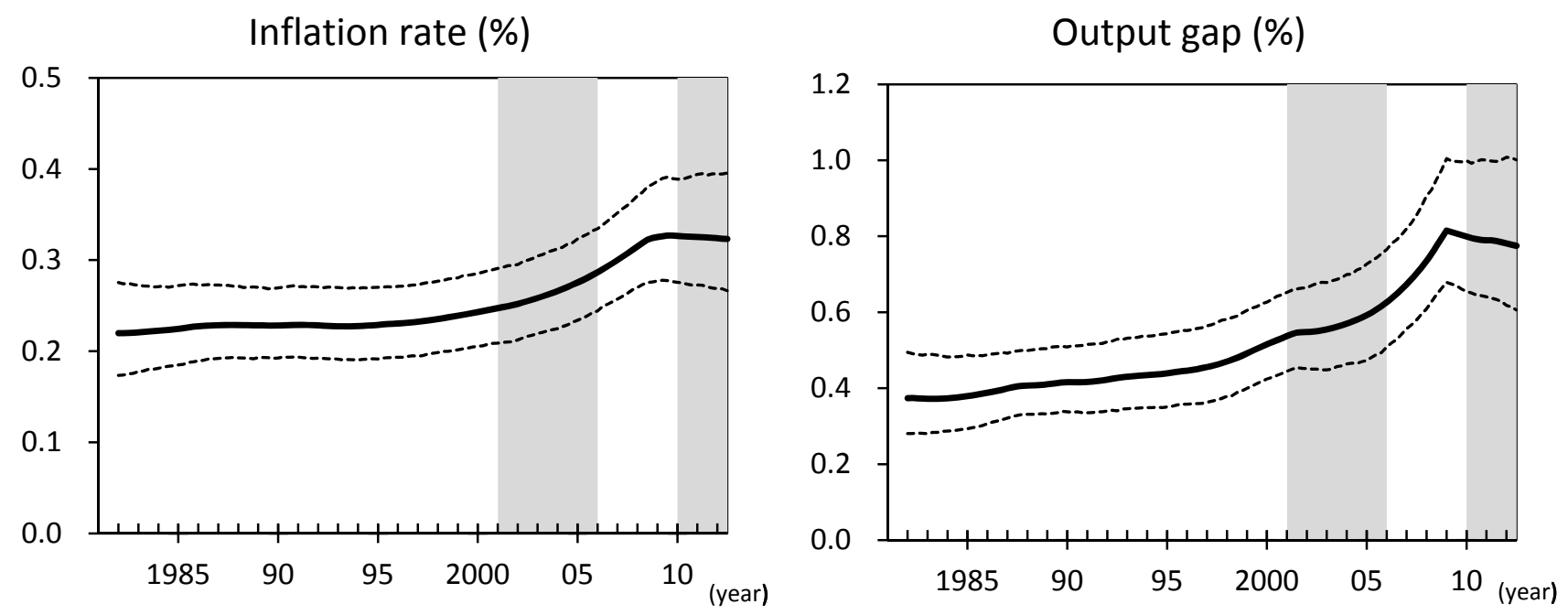

Short-term interest rate (\%)
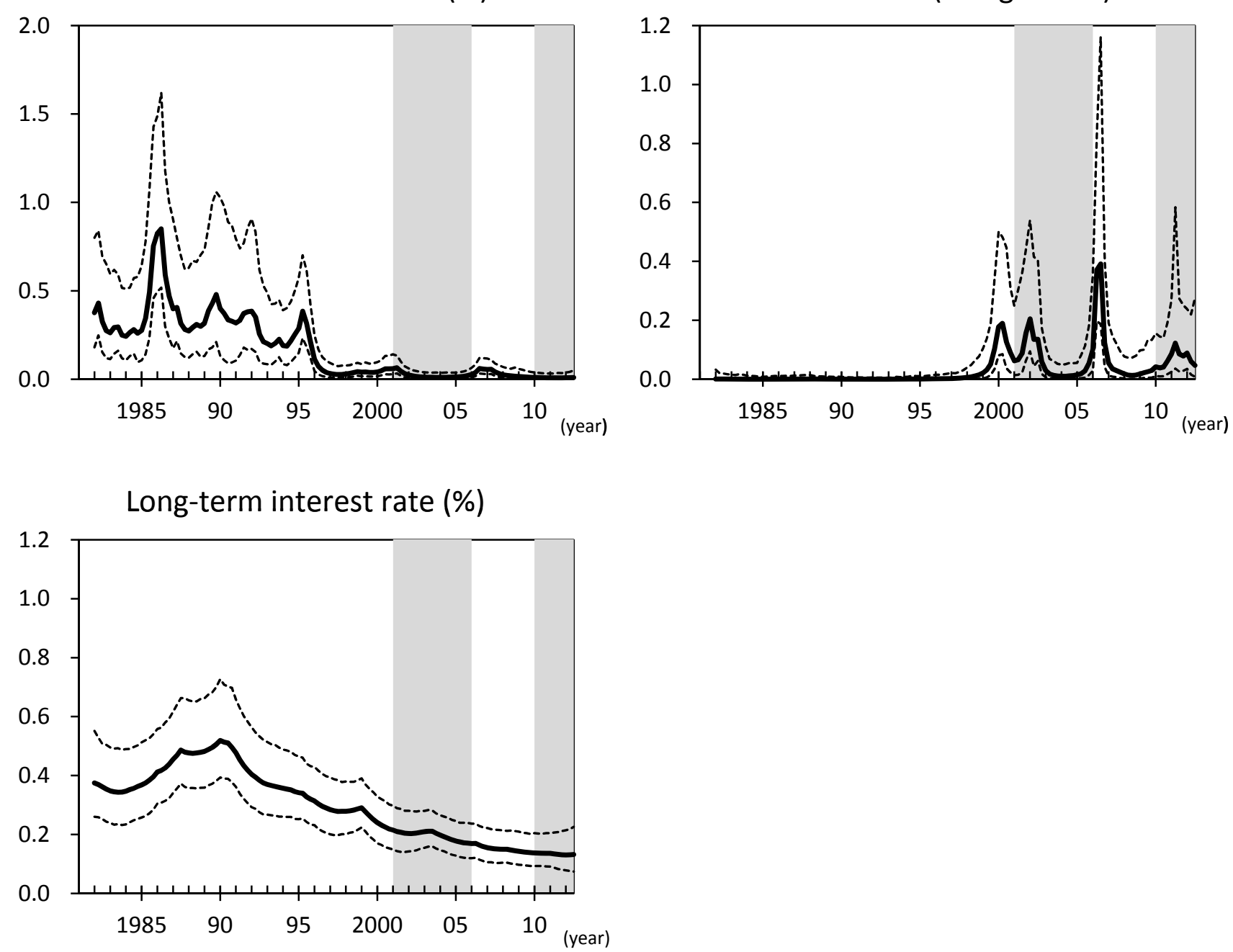

Note: Posterior medians with $68 \%$ credible intervals. 
Figure 6. Impulse responses of policy reaction

(1) Response of short-term interest rate (\%P)
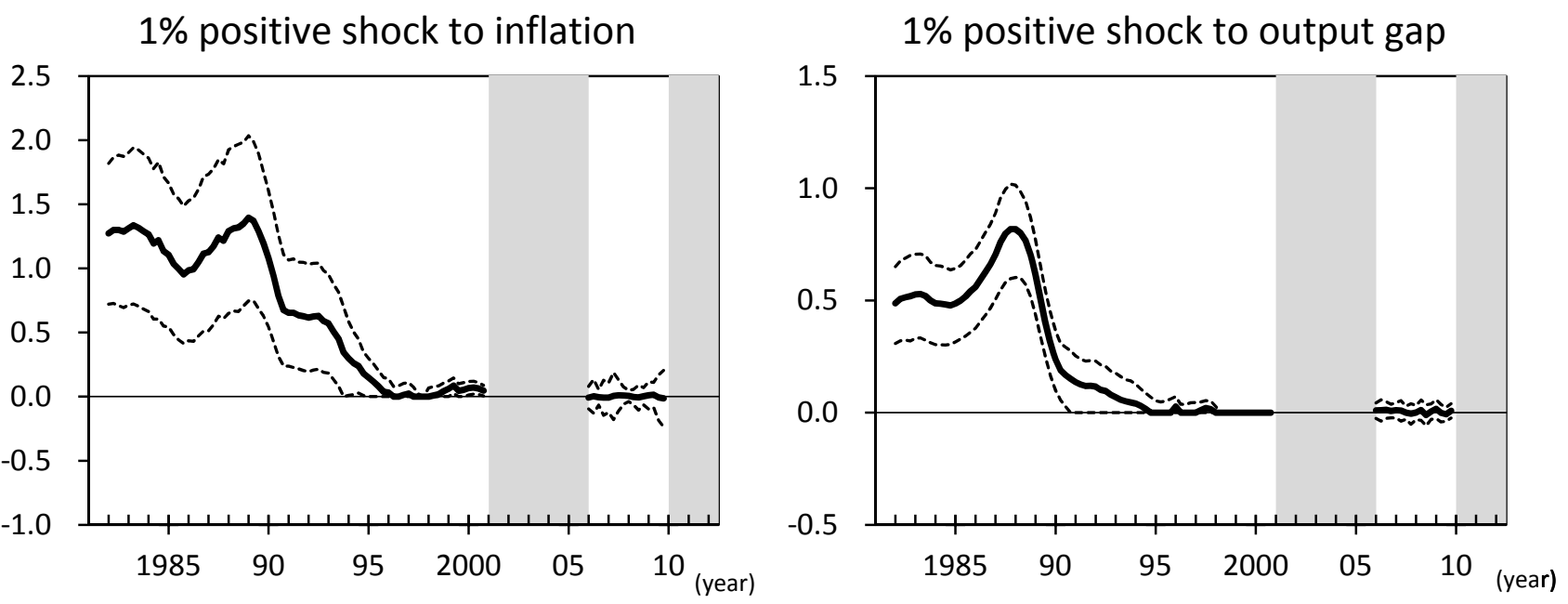

(2) Response of bank reserves (trillion yen)

$1 \%$ negative shock to inflation

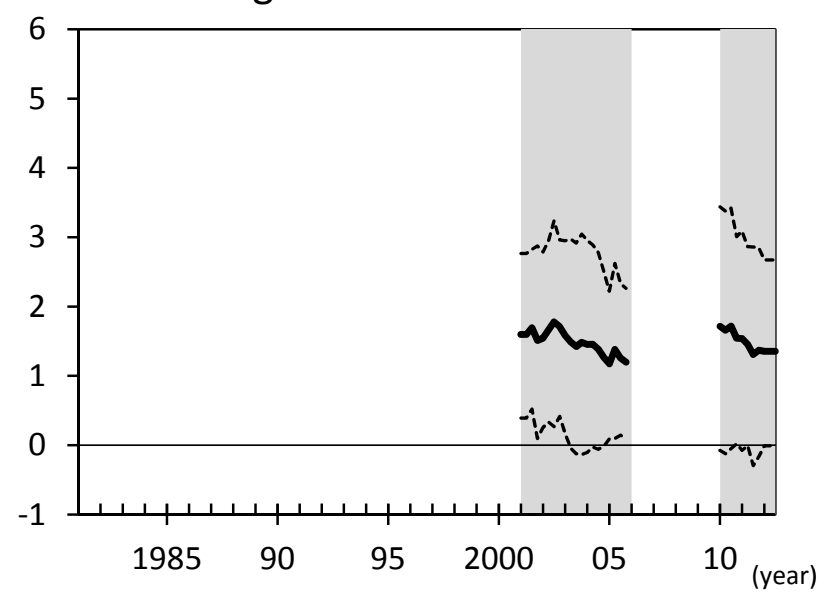

$1 \%$ negative shock to output gap

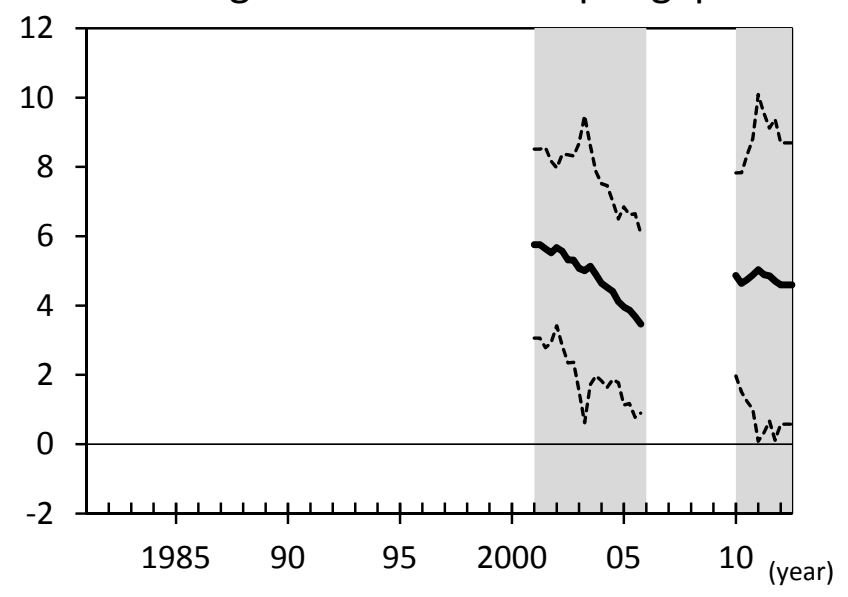

Notes: 1. Responses one year after shock.

2. Posterior medians with $68 \%$ credible intervals. 
Figure 7. Impulse responses to expansionary policy shock

(1) $1 \%$ negative short-term interest rate shock
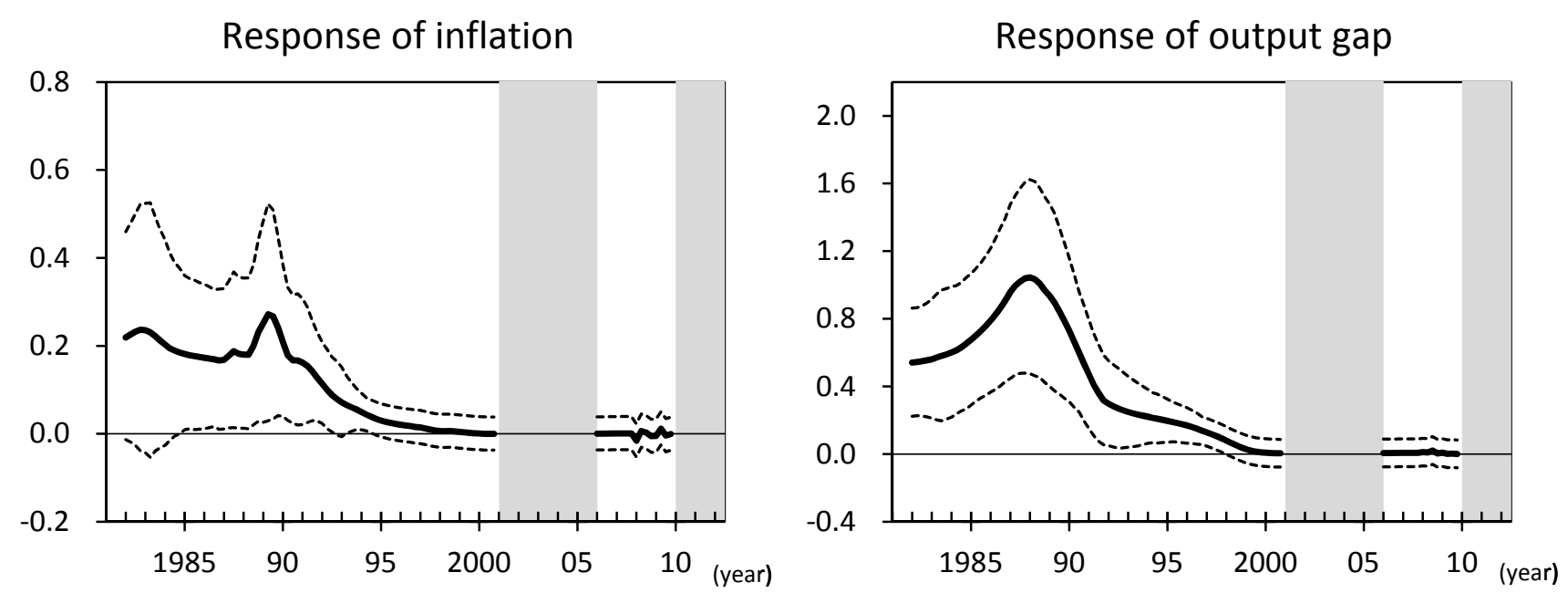

(2) 10 trillion yen positive bank reserves shock
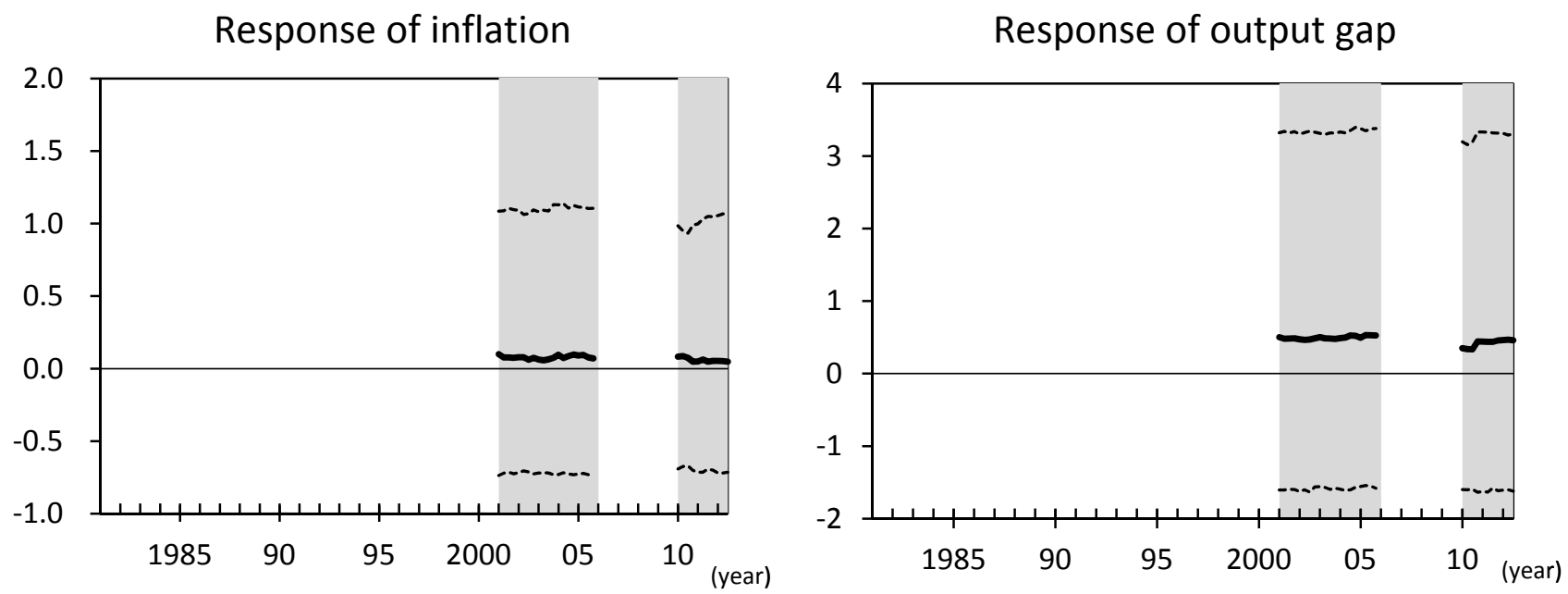

Notes: 1. Responses (\%P) two years after shock for inflation and one year after shock for output gap.

2. Posterior medians with $68 \%$ credible intervals. 
Figure 8 . Impulse responses to $1 \%$ negative long-term interest rate shock
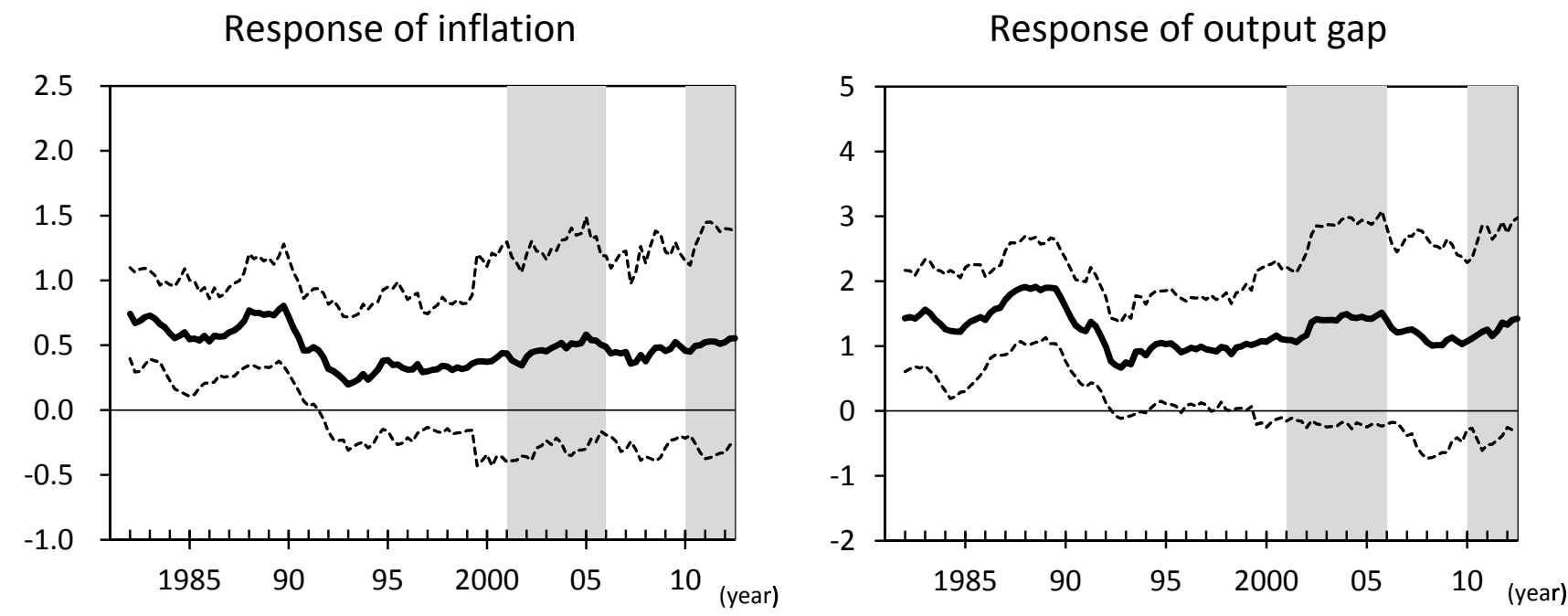

Notes: 1. Responses (\%P) two years after shock for inflation and one year after shock for output gap.

2. Posterior medians with $68 \%$ credible intervals. 
Figure 9. Impulse response of inflation to $1 \%$ positive output gap shock

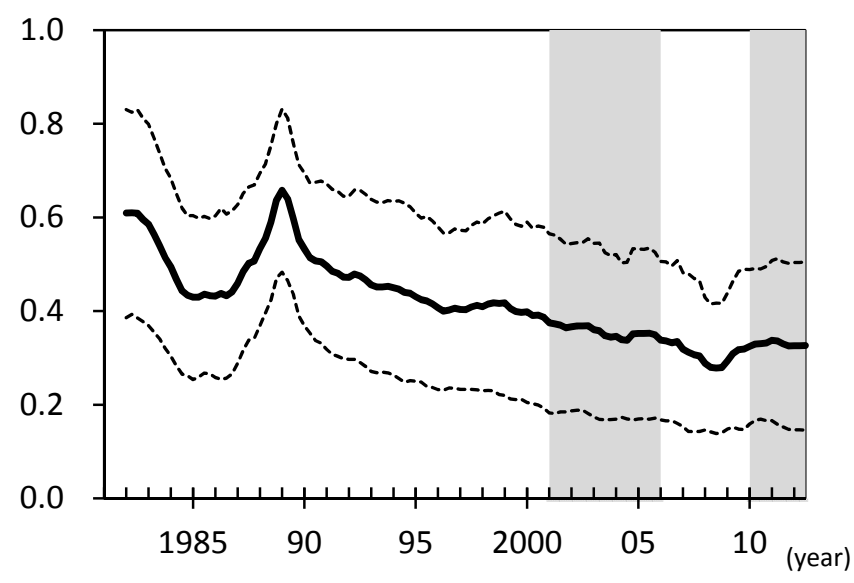

Notes: 1. Response (\%P) two years after shock.

2. Posterior medians with $68 \%$ credible intervals. 